\title{
Machine Learning Trained Face Recognition based Automotive Ignition System
}

\author{
Vamshi Krishna S \\ Associate Professor, \\ Electronics and Communication \\ Department \\ Godavari Institute of Engineering \\ and Technology (Autonomous), \\ Rajahmundry, India
}

\author{
Bharath Kumar M \\ Student, \\ Electronics and Communication \\ Department \\ Godavari Institute of Engineering \\ and Technology (Autonomous), \\ Rajahmundry, India
}

\author{
V S Sai Aravind T \\ Student, \\ Electronics and Communication \\ Department \\ Godavari Institute of Engineering \\ and Technology (Autonomous), \\ Rajahmundry, India
}

\author{
Pavan Kumar P \\ Student, \\ Electronics and Communication Department \\ Godavari Institute of Engineering \\ and Technology (Autonomous), \\ Rajahmundry, India
}

\author{
Naresh Babu V \\ Student, \\ Electronics and Communication Department \\ Godavari Institute of Engineering \\ and Technology (Autonomous), \\ Rajahmundry, India
}

\begin{abstract}
From the past few decades the percentage of individual transportation has increased from $39.2 \%$ to $77 \%$ globally, which shows a rapid growth in the sales of automotive, this also led to an increased rate of stolen vehicles and road accidents. This project aims to introduce an efficient and feasible methodology by implementing face recognition for improved security. The project incorporates image processing where the authorized user's facial features are recognized and verified with the database. Implementation of this system can be done on both motor bikes and motor cars with minimal installation cost which prevents the unauthorized use of vehicles. Keeping in mind the future perspectives, face recognition based automotive ignition system can surely provide a highly secure system thereby avoiding theft and ensuring passenger safety.
\end{abstract}

Keywords-Raspberry-pi, Face Recognition, Ignition System, Open $C V$.

\section{INTRODUCTION}

Automotive theft and accidents are arguably the growing type of crime which are least-solved in our country. While a majority of stolen vehicles are motorcycles, scooters, cars and remaining are heavy vehicles like trucks. The use of Face recognition plays an important role in both security and surveillance which is already being used in different fields, its use and applications are rapidly spreading one of which is the implementation of Machine learning trained face recognition based automotive ignition system, which provides enhanced security and safety to both the user and vehicle.

\section{LITERATURE REVIEW}

In most Literatures Face Recognition and detection has been implemented using various platforms like mat lab, microprocessors, microcontrollers, FPGA etc. [1] They used Raspberry $\mathrm{Pi}$ based face recognition system using conventional face detection and recognition techniques such as Haar cascades. Their main objective is to replace the use of passwords and radio frequency identification cards to access high security systems and buildings with face recognition. [2] They have developed a network that consists a group of computers connected with a microcomputer with a camera that takes images of people, analyse, detect and recognize human faces using image processing and recognition algorithms, such a system can be used as security system in densely populated spots. It can detect and recognize an individual face in numerous situations and scenarios, because it makes use of "Boosted Cascade of Simple Features algorithm" to detect human faces. "Local Binary Pattern algorithm" to recognize these faces. Various other applications of face recognition have been mentioned in literature, [4] home automation using ARMv7 processors with Opencv library and face recognition algorithm based on principal component analysis (PCA). The main aspects of the system are low power consumption, resource optimization and enhanced operation speed.

\section{A. Training mode}

\section{METHODOLOGY}

Training mode, the module is first tuned to run this program where a set of random pictures of the user is captured and saved, these pictures acts like input data. The system makes use of Haar Cascade features and Local Binary Histogram pattern algorithm to train the module for efficient face recognition. 


\section{B. Haar Cascade}

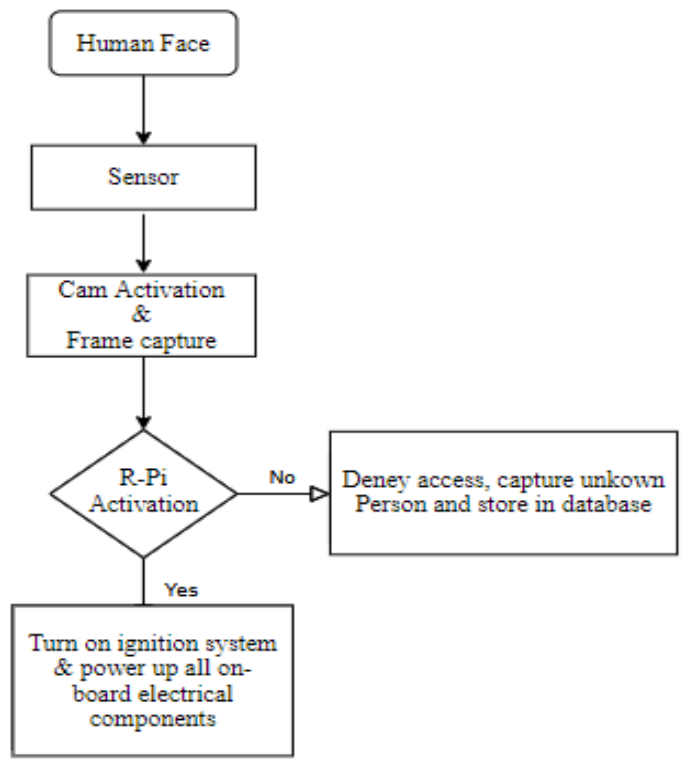

Fig. 1 Steps involved in face recognition

Haar Cascade, an adaptive machine learning based approach where it uses several positive and negative images to train a cascade function which is further used to detect a particular object from images. This algorithm requires more images (face images) to train classifier and extract features from it. Haar-like features are obtained by subtracting sum of pixels under white area from sum of pixels under black area.

$\mathrm{F}($ Haar $)=\sum \mathrm{F}$ white $-\sum \mathrm{F}$ black

$\sum \mathrm{F}$ white $=$ Sum of pixels of bright area

$\sum \mathrm{F}$ black $=$ Sum of pixels of dark area

$\mathrm{F}($ Haar $)=$ the Haar-like feature

\section{C. $L B P H$}

LBPH (Local Binary Pattern Histogram) is a face recognition algorithm, it operates on the texture by labelling the pixels of an image by taking a pixel as the centre and developing threshold of the neighbour pixels value. By comparing neighbour pixels value with the centre pixel value it assigns 1 or 0 to each pixel thus generating a binary value.

$$
\begin{gathered}
\operatorname{LBP}\left(\mathrm{x}_{\mathrm{c}}, \mathrm{y}_{\mathrm{c}}\right)=\sum_{p=0}^{p-} 2^{p} \mathrm{~s}\left(\mathrm{i}_{\mathrm{p}}, \mathrm{i}_{\mathrm{c}}\right) \\
\left(\mathrm{x}_{\mathrm{c}}, \mathrm{y}_{\mathrm{c}}\right)=\text { Centre pixels } \\
\mathrm{i}_{\mathrm{c}}=\text { Centre pixel intensity } \\
\mathrm{i}_{\mathrm{p}}=\text { neighbour pixel intensity }
\end{gathered}
$$

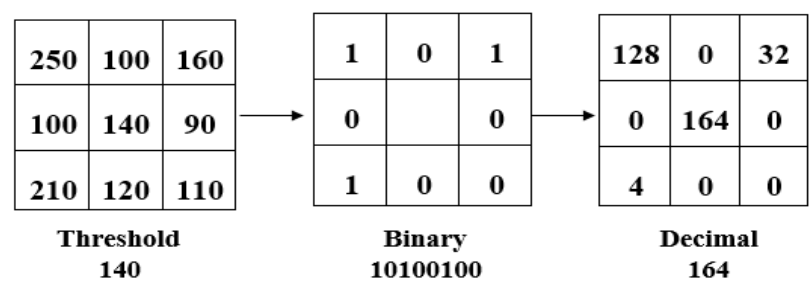

Fig. 2 LBPH operation
- Distance transform is an important tool in image processing, pattern recognition and computer vision as it measures the distance from each object to nearest boundary. In binary images it specifies the distance from each pixel to non-zero pixel. Euclidean distance is the straight-line distance between two pixels and is evaluated using Euclidean norm.

Euclidean distance: $\mathrm{d}(\mathrm{a}, \mathrm{b})=\sqrt{ }(\mathrm{bx}-\mathrm{ax}) 2+(\mathrm{by}-\mathrm{ay}) 2$

- Flow chart gives a brief idea about how the proposed system works, when the sensors detect a human presence it activates the camera which captures the image of human. The captured image frame is then processed to get the region of interest (human face), it is further processed to get the LBPH values using Haar features and then compared with the values of the pre-saved images.

\section{WORKING MECHANISM}

A Raspberry-Pi cam interfaced with the Pi module monitors the surrounding environment when it gets a interrupt signal from the sensors which are installed to detect the human intervention around the vehicle, if the person detected is recognised as a user/owner of the vehicle then the person is granted the access to the vehicle.

A Raspberry-Pi cam interfaced with the Pi module monitors the surrounding environment when it gets a interrupt signal from the sensors which are installed to detect the human intervention around the vehicle, if the person detected is recognised as a user/owner of the vehicle then the person is granted the access to the vehicle.

In fossil fuel Combustion engine based vehicles, on a successful authentication the power supply from battery is allowed to pass to the ignition system which activates spark plugs and ensures the continuous combustion in engine.

In electric vehicles, on successful authentication the power supply from the battery to electric motors or traction motors is allowed for propulsion.

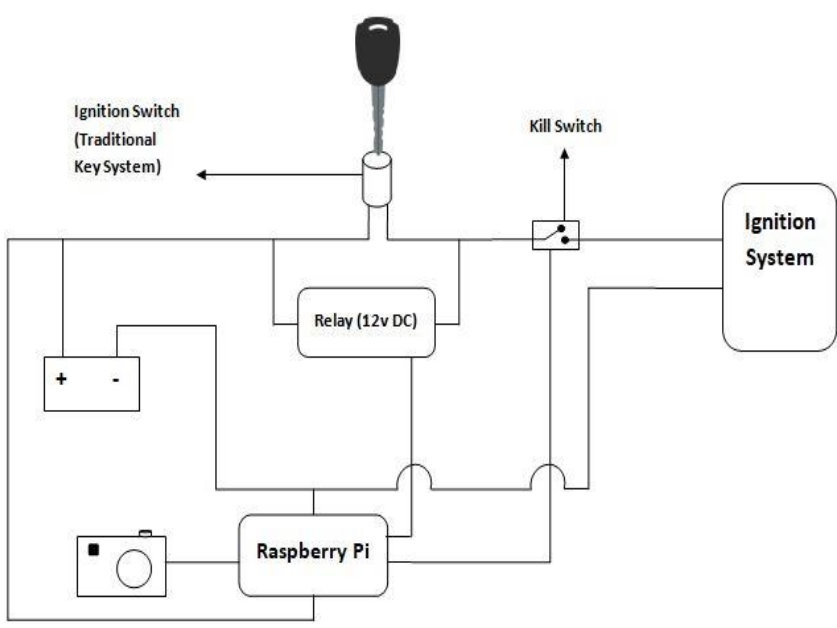

Fig. 3 Diagram of hardware implementation

\section{OUTPUT RESULT}

The result of this project can view in two ways one gives the access and turn on the ignition system and power supply 
and the other denies access when an unauthorised person tries to take control of the automobile.

The system first needs to be trained using a training code to create a data set of the user to verify the user whenever the he appears before the camera. The figure below shows the authorised access when a registered user comes infront of camera the access is granted, power supply and ignition system are turned on. Once the verification is done the system displays face matched for a verified user.

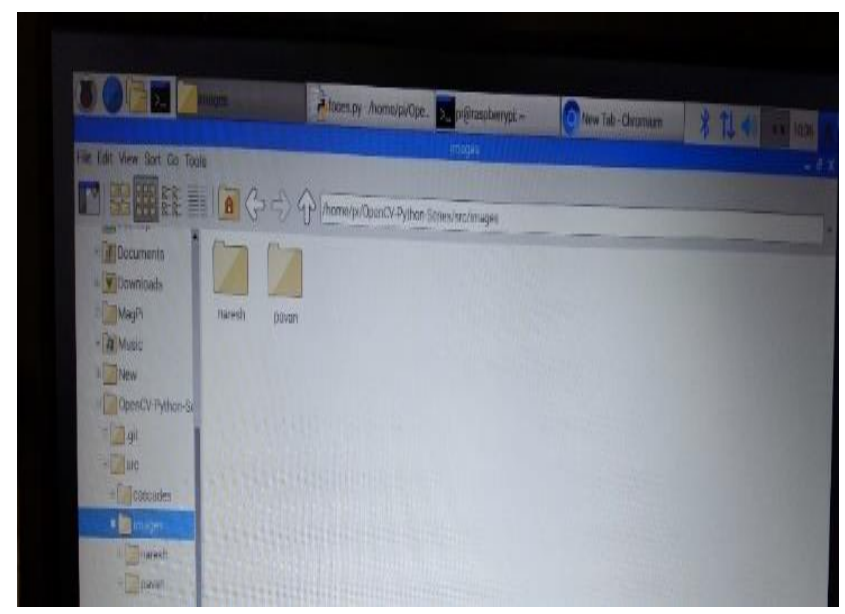

Fig. 4 Data set created during training mode

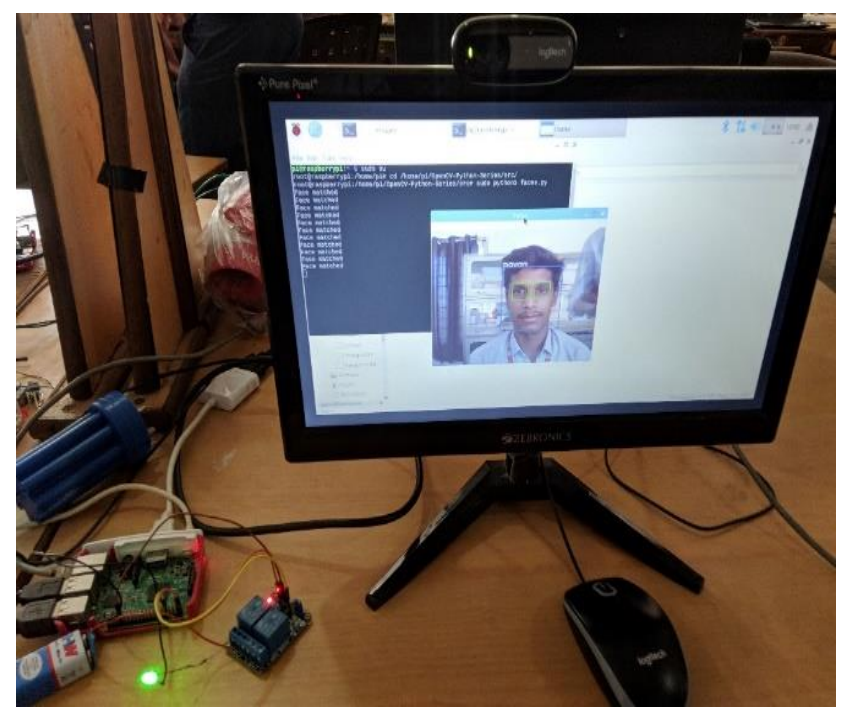

Fig. 5 Shows the authorised access

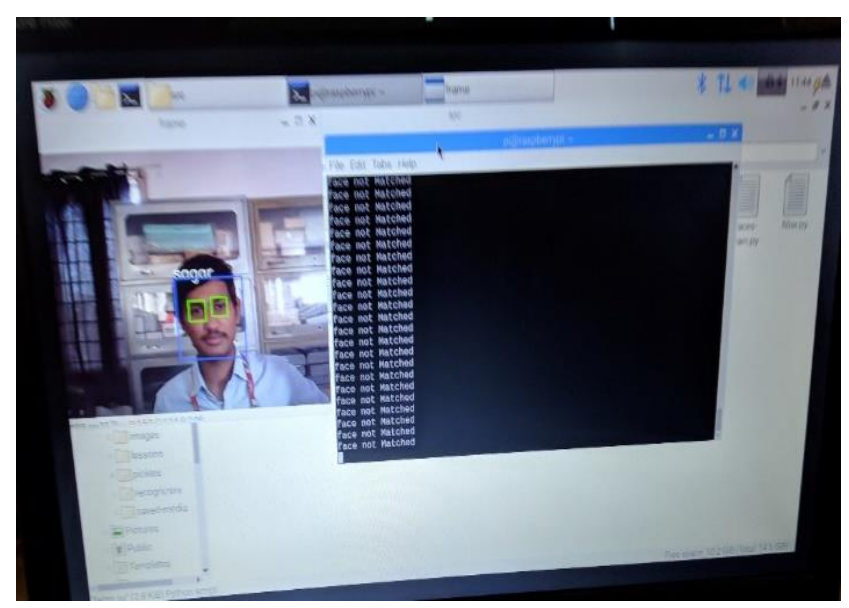

Fig. 6 Shows the unauthorised access
The above figure depicts the unauthorised access when a new person is in front of camera the access is denied, after verification the system shows face not matched text messages.

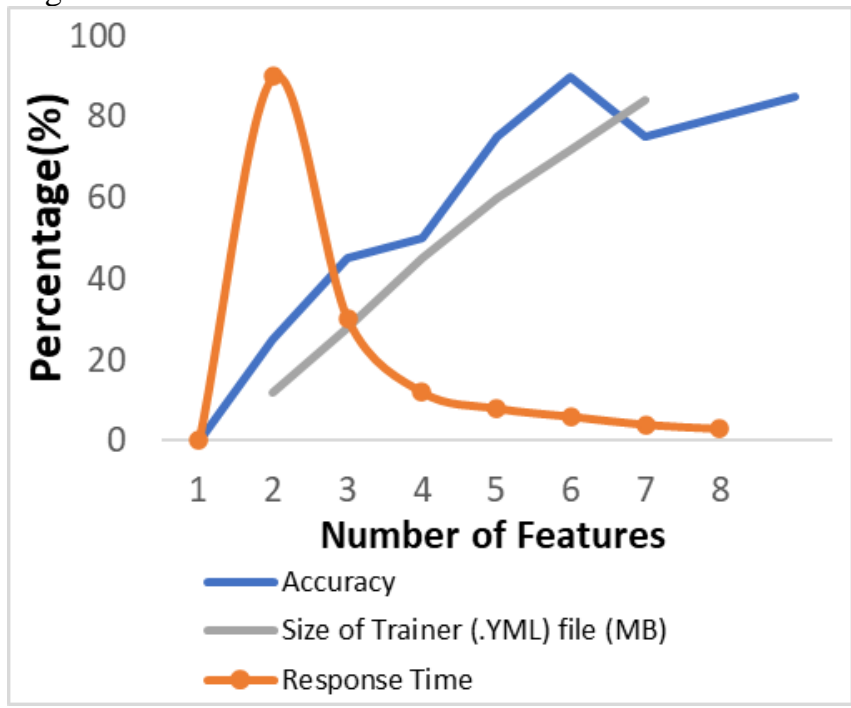

Fig. 7 LBPH plot with different parameters

From our results we have plotted three graphs on different parameters like Accuracy, size of trainer file and response time using Local Binary Pattern Histogram algorithm.

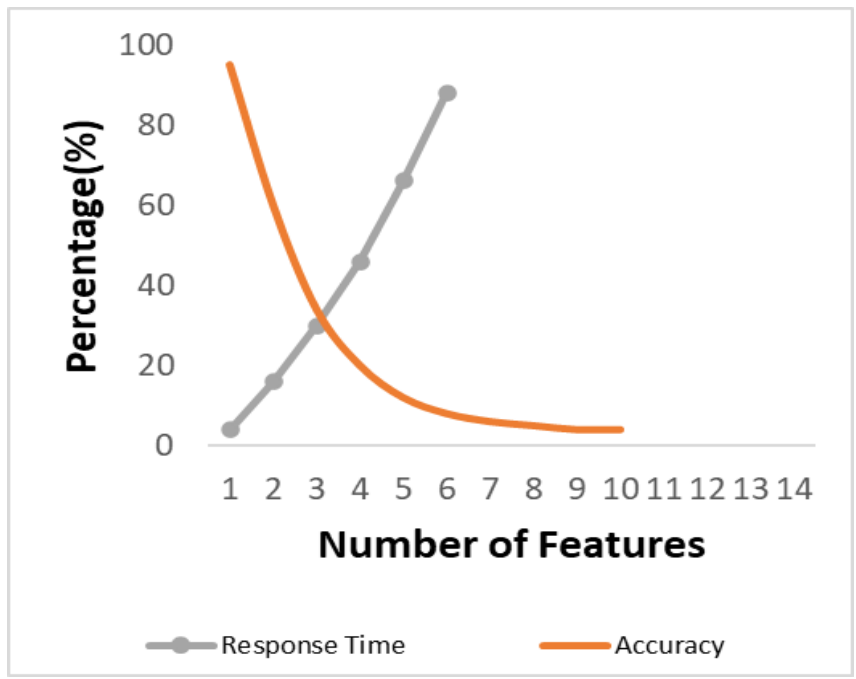

Fig. 8 Response time and accuracy of eigen faces

The above graph is plotted using Eigen faces algorithm on parameters like response time and accuracy. 


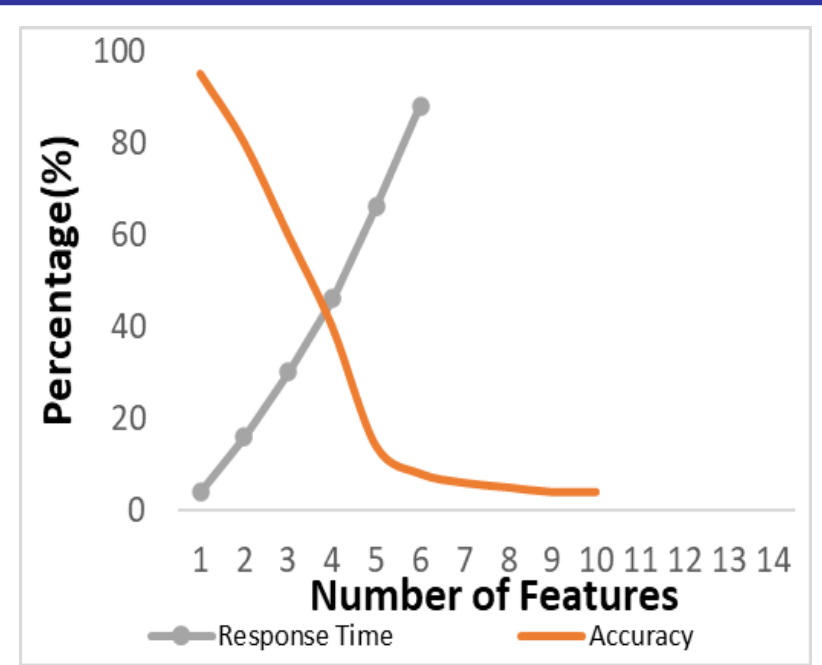

Fig. 9 Response time and accuracy of fisher faces

Above graph is plotted using Fisher faces algorithm on parameters like response time and accuracy

By comparing all the three graphs on different face algorithms we can conclude that Local Binary Pattern Histogram algorithm is considered as efficient algorithm for face recognition and detection.

\section{CONCLUSION}

This paper aims to propose system which is compact in size and can be fitted in the automotive like two wheelers, four wheelers and heavy vehicles. As this system makes use of Machine Learning tools so the various problems like ageing factor, pose variations etc can be overcome. With the addition of few sensors and code lines, this system can also alert the user in an intrusion case and can be used to pre-warn the driver about the chance of collision by measuring the distance between the vehicles.

\section{ACKNOWLEDGMENT}

We would like to express our gratitude for help provided by our college Godavari Institute of Engineering and Technology authority for helping us to develop this paper.

\section{REFERENCES}

[1] Ishita Gupta, Varsha Patil, Chaitali Kadam and Shreya Dumbre, "Face Detection and Recognition using Raspberry Pi", IEEE - 2016 International WIE Conference on Electrical and Computer Engineering, WIECON-ECE, December-2016.

[2] Ayman A. Wazwaz, Amir O. Herbawi, Mohammad J. Teeti and Sajed Y. Hmeed, "Raspberry Pi and Computers-Based Face Detection and Recognition System", 4th International Conference on Computer and Technology Applications, June-2018.

[3] Ketan J. Bhojane, S.S. Thorat, "Face Recognition Based Car Ignition and Security System", International Research Journal of Engineering and Technology (IRJET), e-ISSN: 2395-0056, May-2018.

[4] N. Stekas, D. Heuvel, "Face Recognition Using Local Binary Patterns Histograms (LBPH) on an FPGA-Based System on Chip (SoC)", IEEE, Chicago, IL, USA, Aug-2016.

[5] Bhaumik Vaidya, Ankit Patel, Anand Panchal, Rangat Mehta, Krish Mehta and Parth Vaghasiya, "Smart home automation with a unique door monitoring system for old age people using Python, Open CV, Android and Raspberry pi”, International Conference on Intelligent Computing and Control Systems (ICICCS), July-2017.

[6] Neel Ramakant Borkar and Sonia Kuwelkar, "Real-Time Implementation Of Face Recognition System", IEEE - 2017 International Conference on Computing Methodologies and Communication, July-2017.

[7] Gagandeep Singh, Jappreet Singh and Nishant Yadav, "Facial Detection and Recognition using OpenCV on Raspberry Pi Zero", International Conference on Advances in Computing, Communication Control and Networking (ICACCCN), Oct-2018.

[8] Teddy Mantoro, A. Ayu and Suhendi, "Multi-Faces Recognition Process Using Haar Cascades and Eigenface Methods", IEEE, May2018.

[9] Kirti Dang and Shanu Sharma, "Review and Comparison of Face Detection Algorithms", IEEE, Jan-2017. 